\title{
Magneto-elastic Bilayers for Sleep Apnea Monitoring
}

\author{
S. Traxler ${ }^{a}$, H. Pfützner, E. Kaniusas, K. Futschik \\ Institute of Electrodynamics, Microwave and Circuit Engineering (EMCE), \\ Vienna University of Technology, Gusshausstrasse 25/354, 1040 Vienna, Austria \\ atraxler@mail.tuwien.ac.at
}

\begin{abstract}
Keywords: Magneto-elasticity, Artificial Neural Network, Obstructive Sleep Apnea Syndrome, Curvature Sensor.
\end{abstract}

\begin{abstract}
Magneto-elastic bilayers (BLs), consisting of a magnetostrictive layer and a non-magnetic counter layer, show highest sensitivity with respect to bending. This paper describes a biomedical application in the field of sleep apnea screening. A multi-parametric detector fixed at the thorax contains two BLs. One BL yields a skin curvature sensor adjusting itself to curvature variations given by physiological activities. The second BL exhibits a free end thus working as a motion sensor. The two signals are fed into artificial neural networks for the detection of events like normal respiration and apneas, as well as body movements and position.
\end{abstract}

\section{Introduction}

Some percent of the population - i.e., an extremely large number of individuals - suffer from the so-called obstructive sleep apnea syndrome. Individuals suffering from this syndrome have an air passage closure that leads to cessation of respiration during sleep. If the cessation time exceeds $10 \mathrm{~s}$, an apnea is given by definition. Two kinds of apnea are distinguished. If the cessation of respiration is caused by the absence of stimulus for respiration from the central nervous system, it is called a central apnea (CSA). If respiration effort is given but caused by obstruction, the effective airflow is lower than $20 \%$ of normal, it is called an obstructive apnea (OSA).

A few apneas a night are quite normal, but high numbers during the sleep may cause severe health problems. Beside daily sleepiness and stress, an increased blood pressure or micro-sleep attacks can reduce life expectation.

Full assessment of the obstructive sleep apnea syndrome is performed in (rare) sleep laboratories. A so called polysomnography includes at least an electroencephalogram, an electrooculogram and an electromyogram. The measurement of the respiration volume and/or airflow and an electrocardiogram is standard.

Faster and less costing screening tests are made by home monitoring devices. However, they tend to comprise several detectors which yield complicated attachment, and they impair the quality of sleep.

The present work aims on a single detector which contains several sensors for multi-parametric information. The sensors are embedded in a soft shell, which are attached to the chest using a skin friendly adhesive. The present paper is focused on two sensors:

(i) A skin curvature sensor (SCS) which should detect respiratory activity, and

(ii) a motion sensor (MS) which should detect motions and position changes of the body.

A key element of the present sensor concept is given by magneto-elastic bilayers (BLs). As closer described in [1-3], a BL consists of a magnetostrictive layer (ML) which is attached to a nonmagnetic counter layer (CL).

If the $\mathrm{BL}$ is bent to a curvature $c=1 / r$ (with $r$ as the radius) according to Fig. 1, the CL gets under tensile stress $(\sigma>0)$. On the other hand, the ML is compressed $(\sigma<0)$ throughout its cross section. This yields a decrease of permeability $\mu$ as a basis of signal establishing. The latter is performed by a coil excited with a frequency below $15 \mathrm{kHz}$, thus restricting eddy currents in the 
ML. Compared to a bent single magneto-elastic layer, the curvature sensitivity is increased by factors up to 50 [1].

\section{Detector Design}

The ML can be made of any magnetostrictive material. The CL material has to be chosen so that the change of permeability $\mu$ in the ML is not counter-acted by changes of $\mu$ in the CL. That means that the CL can be made of any non-magnetic material and even of magnetic material with much lower magnetostriction, or magnetostriction of different sign. Its mechanic properties are also important. A too soft material would not apply much stress on the ML. On the other hand, a too stiff one would make the whole sensor stiff which is especially disadvantageous for biomedical application where the BL should adjust its curvature to soft tissue, as given for the skin curvature sensor (SCS).

Finally for the given task, amorphous Fe-based Vitrovac with a thickness of $25 \mu \mathrm{m}$ was used for the ML. It has the advantage of high saturation magnetostriction of $\lambda_{\mathrm{S}}=24-35 \mathrm{ppm}$. Also, it is commercially available as thin ribbons, which is advantageous for low cost sensors that are planned in high quantity. From all the possibilities, Vitrovac in combination with non-magnetic steel of $45 \mu \mathrm{m}$ has been chosen because this type of BL yields very high sensitivity for bending [1]. The elasticity modules are $150 \mathrm{GPas}$ for Vitrovac and $180 \mathrm{GPas}$ for the non-magnetic steel. The two layers were glued together with UHU-Endfest 300 with an elasticity module of 1 GPas.

The concept of the bilayer sensor can be used in several ways. Two possibilities are demonstrated within this paper. As already mentioned the first concerns the measurement of skin curvature changes due to physiological activities. The second uses the BL as an acceleration sensor for the registration of body movements similar to [4] but aiming for movements and body position rather than cardiac activity.

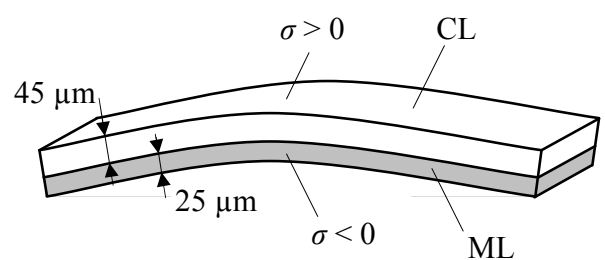

Fig. 1: Basic concept of a bilayer (BL) consisting of a magnetic layer (ML) and a counter layer (CL). The depicted case of bending yields tension of the CL and compression of the ML which is used to establish a sensor signal.

Fig. 2 summarizes the total of experimental arrangement. Two sensors are connected to measurement electronics, which produce a voltage proportional to the impedance of the measurement coil. A manual offset adjustment of the measurement amplifier allows the compensation of the constant part of the measurement coil's impedance to improve the resolution after digitalization.

For biocompatibility and softness, the detector is capsuled by silicon rubber and mounted on the skin of the chest by adhesive tape. It was positioned in front of the breastbone (sternum) in cardiac region. The two contained sensors are based on BLs of about $40 \mathrm{~mm} \times 7 \mathrm{~mm} \times 0.1 \mathrm{~mm}$ size, exhibiting the following features:

The skin curvature sensor (SCS) - It is given by a BL that is mounted directly above the tape. Thus its curvature $c$ adjusts itself to $c$ of the skin. In order to attain a representative average over the BL length, the winding of about 200 turns covers the BL in a homogeneous way. When $c$ of the chest changes, e.g. caused by respiration, also the curvature of the sensor changes, resulting in mechanical stress within the BL, which leads to the signal.

The motion sensor (MS) - Here one end of the BL is fixed on a holder. The second free end carries a small mass $m=0.2 \mathrm{~g}$. The curvature - controlled by gravity + inertia - shows a local 
maximum $c_{\max }$ close to the holder corresponding to maximum change of $\mu$ in this region. For high detection sensitivity, the winding is concentrated here. A trade-off between sensitivity and resonance frequency is given. A higher mass $m$ will increase the curvature caused by gravity and inertia, but it also lowers the resonance frequency and causes more oscillation artifacts. The BL length $l$ has a similar influence. The longer the BL the higher the torque and thus the curvature at a given $m$ and acceleration. From a simple cantilever model we know that it is better to increase $m$ than increasing $l$. This is because both linearly increase the torque $\tau=m \cdot a \cdot l$ with $a$ as the acceleration of the sensor, but the length decreases the resonance in the power of minus three halfs while the mass only decreases it in the power of minus one half according to the formula: $f_{\mathrm{R}} \sim 1 / \sqrt{m \cdot l^{3}}$.

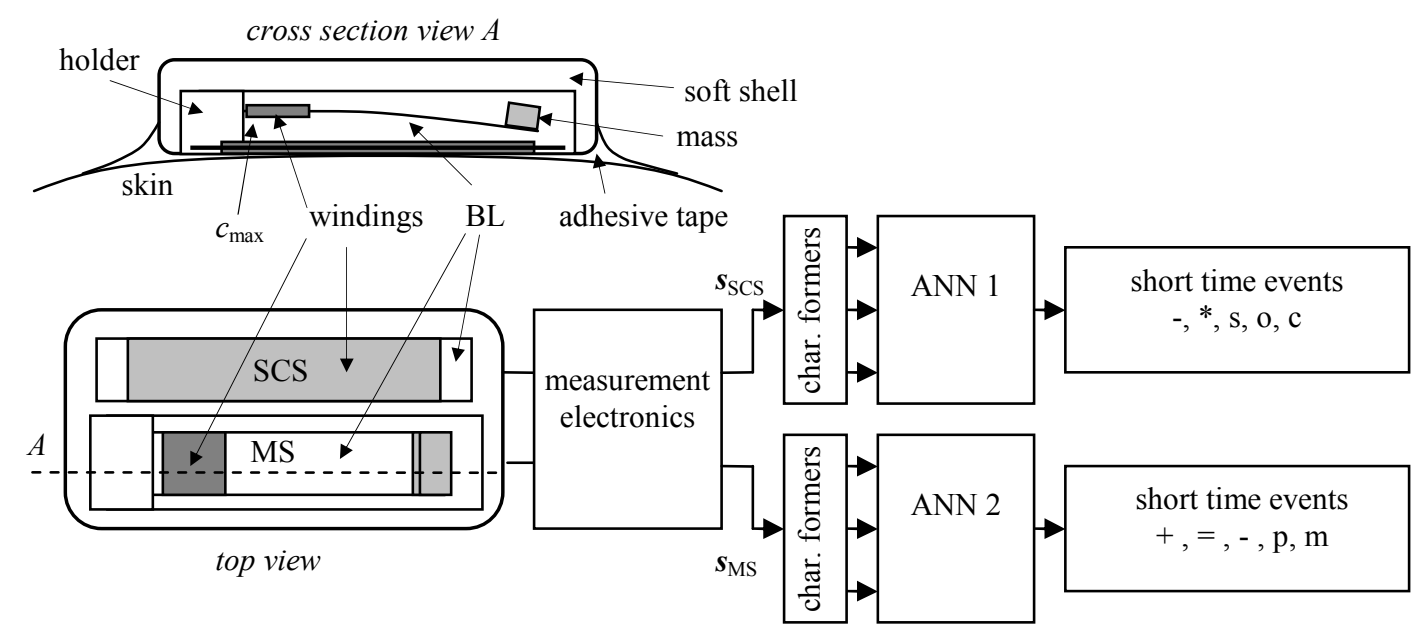

Fig. 2: The detector includes a skin curvature sensor (SCS) and a motion sensor (MS). The signals are processed by characteristics formers and artificial neural networks for event recognition.

However, the model of a simple cantilever may not be very accurate because the BL consists of three layers. Measurement results show that the assumption of a simple cantilever is at least justified for a first order estimation.

\section{Sensor Performance}

Fig. 3a shows a typical signal $s_{\mathrm{SCS}}$ from the skin curvature sensor. The five minute long signal shows periods of normal breathing as well as simulated apneas of different kind, and snoring.

For time windows of $10 \mathrm{~s}$, short time events are indicated by the following symbols: "-" means that normal respiration is given. "*" means normal snoring as a second negative finding. As a positive finding "s" means obstructive snoring. "o" indicates an OSA event characterized by a distorted signal of low amplitude, "c" a CSA event of ceasing respiration due to lacking neural stimulation from the brain.

Fig. $3 \mathrm{~b}$ shows a 10 minutes long signal $s_{\mathrm{MS}}$ of the motion sensor. It displays body positions and the related movements. Again there are short time events with a duration of 10 s indicated. "+" indicates that the patient is in back position which favors OSA. "-" and "=" mean abdominal position and side position, respectively, both favoring negative diagnostics. The ripple represents respiratory activity. Position changes " $p$ " are characterized by sharp signal jumps. "m" means motion, indicating that awake state is given if arising in irregular continuation. 


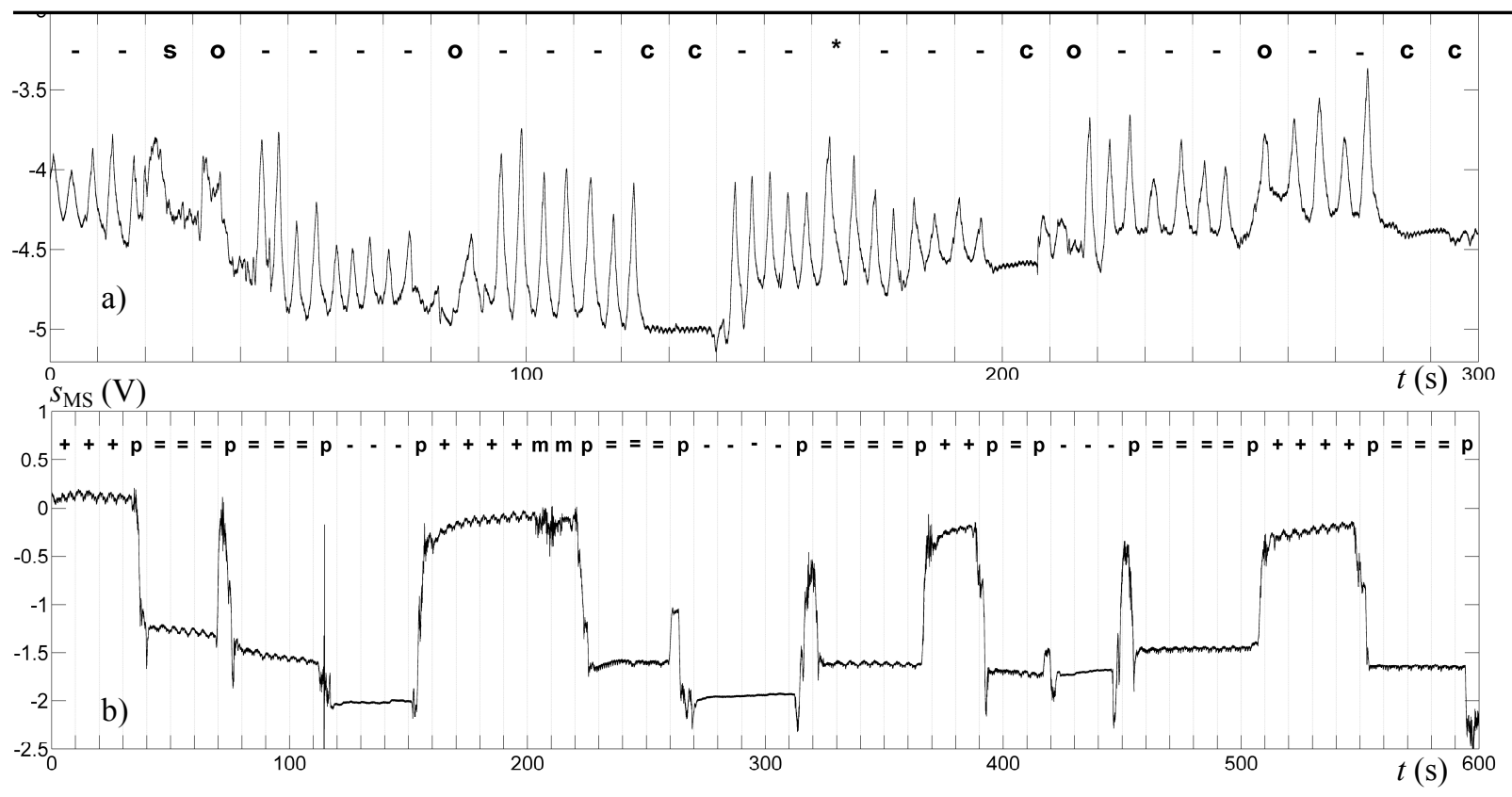

Fig. 3: Examples of sensor signals.

(a) Skin curvature sensor: normal breathing '-', snoring normal and obstructive '*6, 's', obstructive apnea 'o', central apnea ' $c$ '

(b) Motion sensor: back position '+', side position '=', abdominal position '-', position change 'p', movement 'm'

Fig. 2 also includes the evaluation of sensor signals. The corresponding description is given in Chapter 4. The signals are fed in characteristic formers, followed by artificial neural networks (ANNs) for the recognition of short time events for time windows of $2 \mathrm{~s}$ up to $10 \mathrm{~s}$.

\section{Signal Evaluation}

Evaluation of the skin curvature sensor signal $s_{\mathrm{SCS}}$ was performed offline in three major steps. Step 1 was filtering and windowing. The signal was low pass filtered with a cut off frequency of $2 \mathrm{~Hz}$, the mean value was removed and its length was adjusted to a multiple of the window length. For the results of this paper, a window length of $10 \mathrm{~s}$ has been chosen to include at least one cycle of respiration.

Step 2 was the establishment of signal characteristics. Non-specific features included the following:

(i) the RMS value $s_{\text {RMS }}$,

(ii) the peak-to-peak value $s_{\mathrm{PP}}$,

(iii) the mean value $s_{\text {mean }}$, and

(iv) the related RMS factor $F_{\mathrm{REL}}=s_{\mathrm{RMS}} / s_{\text {mean }}$.

Specific features were defined with respect to increased distortion for obstructive apnea events:

(v) the windows pixel density $D_{\mathrm{P}}$ defined as the number of pixels as contained in the signal $s$,

(vi) the harmonics factor $F_{\mathrm{H}}$ defined as the ratio of the high frequency $(>1 \mathrm{~Hz})$ filtered signal $s_{\mathrm{HF}, \mathrm{PP}}$ and the low frequency $(<1 \mathrm{~Hz})$ filtered signal $s_{\mathrm{HF}, \mathrm{PP}}$.

Step 3 was to feed the six features into a feed forward ANN with 6 input neurons, 4 neurons in the hidden layer and 4 output neurons for the events -, o, c and "not classified" for everything else (e.g. movement artifacts) according to Fig.2 (not aiming for snoring events * and s). Later, a microphone will be used for the registration of the latter.

First attempts of training were performed with data from about 1 hour of simulated sleep. Testing through the same data set yielded the following results:

15 obstructive apnea events "o" - 12 detected correctly, 3 classified as "c" (central apnea event) and "-" (normal respiration);

33 central apnea events "c" - 32 detected correctly, 1 as normal respiration "_.";

243 normal respiration "_. -241 detected correctly, 1 as "o" and 1 as "c". 
As a conclusion, the procedure is promising, but problems have to be overcome with respect to obstructive events which are of high significance.

Evaluation of the signal $s_{\mathrm{MS}}$ was performed in an analogous way. But filtering was performed with $10 \mathrm{~Hz}$, and the features were restricted to $s_{\mathrm{HF}, \mathrm{PP}}$ and $s_{\text {mean }}$. Consequently, the ANN was restricted to 2 input neurons, 4 hidden ones and 4 output neurons corresponding to,$+=,-$, and $\mathrm{p} / \mathrm{m}$ according to Fig.2. In general, correct classification was attained with results as indicated in Fig.3b. About $15 \%$ of events were classified in erroneous ways. However this was restricted to windows close to position changes thus being without practical relevance. Also here, data was used from simulation of sleep which however can be assumed to be negligible for the case of $s_{\mathrm{MS}}$.

\section{Conclusions}

For sleep apnea monitoring, a novel detector was developed containing two sensors based on magneto-elastic bilayers (BLs). For the given task, good performance was found for amorphous Vitrovac agglutinated on non-magnetic steel. This yielded sufficient curvature sensitivity in combination with rather low temperature sensitivity. A skin curvature sensor SCS detects respirationcaused curvature changes of the skin. A motion sensor MS was operated in acceleration mode thus also detecting global position changes of the body.

Signal evaluation was based on 10 s short time events. They were fed into two artificial neural networks trained with results from simulated sleep. For SCS, apnea events were classified in a promising way, however improvements being necessary with respect to obstructive apnea events. For MS, satisfying classification was attained.

The next tasks will concern testing in clinical surrounding. However, the so far results indicate a promising system performance. The two sensors yield a high amount of information as a basis for the recognition of apneas of different types. As an important advantage, both sensors use a common technical concept. BLs are robust, extremely light (order of $1 \mathrm{~g}$ ), flat and cheap. This may yield a screening tool which allows wide routine inspection, including applications in the $3^{\text {rd }}$ world.

\section{Acknowledgment}

We thank for support from the science funds FWF (Project No. P19886).

\section{References}

[1] H.Pfützner, E.Kaniusas, J.Kosel, L.Mehnen, T.Meydan, F.Borza, M.Vazquez, M.Rohn, A.M.Merlo, B.Marquardt, J. Mater. Proc. Technol., 181, pp. 186-189, (2007).

[2] H.Pfützner, E.Kaniusas, J.Kosel, L.Mehnen, T.Meydan, M.Vázquez, M.Rohn, A.M.Merlo, B.Marquardt, Sensors and Actuators A: Physical, 129, pp. 154-158, (2006).

[3] S.Traxler, J.Kosel, H.Pfützner, E.Kaniusas, L.Mehnen, I.Giouroudi: Sensors and Actuators A: Physical, 142, pp. 491-495, (2008).

[4] Kaniusas, L.Mehnen, C.Krell, H.Pfützner, J. Magn. Magn. Mater., 215-216, pp. 776-778, (2000). 